\title{
STABILITY ANALYSIS OF MIXED FINITE ELEMENT FORMULATIONS WITH SPECIAL MENTION OF EQUAL-ORDER INTERPOLATIONS
}

\author{
S. IDELSOHN, M. STORTI AND N. NIGRO \\ Grupo de Tecnologia Mecánica del INTEC, CONICET, Universidad Nacional del Litoral, Güemes 3450, 3000 Santa Fe, \\ Argentina
}

SUMMARY

We present a method to assess the stability of pairs of interpolation spaces for mixed formulations. The method is based on a straightforward calculation of the eigenvalues of the discrete matrices through Fourier decomposition in plane waves and is intended to give, via straightforward numerical computations, a sharper determination of stability than the well-known 'patch test' of Zienkiewicz et al. Special attention is devoted to the study of stability and accuracy of equal-order interpolations.

KEY WORDS incompressible flow; mixed formulations; stabilized algorithms; Fourier analysis; finite elements

\section{INTRODUCTION}

Mixed formulations arise commonly in the approximation of physical problems with 'internal restrictions' as in incompressible fluid mechanics or elasticity, plate theory and the Maxwell equations, as well as from the need to improve the convergence order for the derivatives of the independent variables as in the stress-displacement formulation of elasticity. The numerical solution of such problems involves the particular difficulty of finding the correct pair of interpolation spaces for the 'primary variables' and the 'constraint variables'. A sufficient condition for a convergent approximation is the satisfaction of the so-called Brezzi-Babǔska or 'inf-sup' condition. However, the assessment of whether a given pair of spaces satisfies this condition or not has proved not to be an easy task. Zienkiewicz et al. ${ }^{1}$ proposed a simple algebraic test, the 'patch test', based on a straightforward counting of degrees of freedom. This test discards from the outset a lot of a priori admissible interpolations. However, the extension to more complex problems, e.g. the stress-velocitypressure formulation which is at the basis of viscoelastic flow modelling, ${ }^{2,3}$ is not evident, mainly with respect to what kind of boundary conditions have to be enforced at the boundary of the patch.

Instability of a given interpolation is associated with the existence of 'spurious checker-board modes', which are high-frequency pressure modes (speaking of the velocity-pressure formulation of the Stokes equations) which are 'not seen' by all the admissible (approximately incompressible) velocity fields. These modes could be eliminated from the pressure space, but it happens that those modes which are very near (in frequency) to them have a small 'stability quotient' (the quotient in the inf-sup condition) and this quotient is not uniformly bounded from below with respect to the mesh parameter, as it should be to guarantee a convergent approximation. We follow here the discussion about the Q1/P0 element (quadrangles with bilinear continuous velocities and constant discontinuous

CCC 0271-2091/95/091003-20

(C) 1995 by John Wiley \& Sons, Ltd. 
pressures) in Chapter 1, $\$ 3.3$. of Reference 4. In that reference the checker-board mode is identified and it is shown that even upon eliminating it from the pressure space, the inf-sup condition is not uniformly satisfied. It is shown there that the condition is not satisfied by an element of the pressure space which is basically the high-frequency checker-board mode modulated by a smooth envelope (a 'wave packet'). This reasoning led us to contemplate the possibility of assessing stability by Fourier analysis of the matrices involved.

In this work we develop an algebraic criterion to assess stability by straightforward calculation of all the eigenvalues for periodic problems on (more or less) regular meshes. The criterion is then applied to all the family of discontinuous and continuous prssure interpolations. A detailed study of stability and precision is given for stabilized algorithms.

\section{THE BREZZI-BABǓSKA CONDITION FOR STOKES FLOW}

Consider the Stokes flow

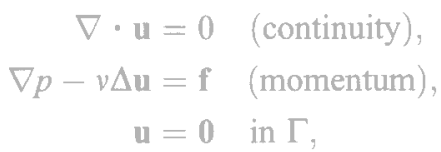

where $\mathbf{u}$ is the velocity vector, $p$ is the pressure, $v$ is the kinematic viscosity and $\mathbf{f}$ is a body force source term. For simplicity we assume homogeneous Dirichlet boundary conditions. The corresponding weak form is
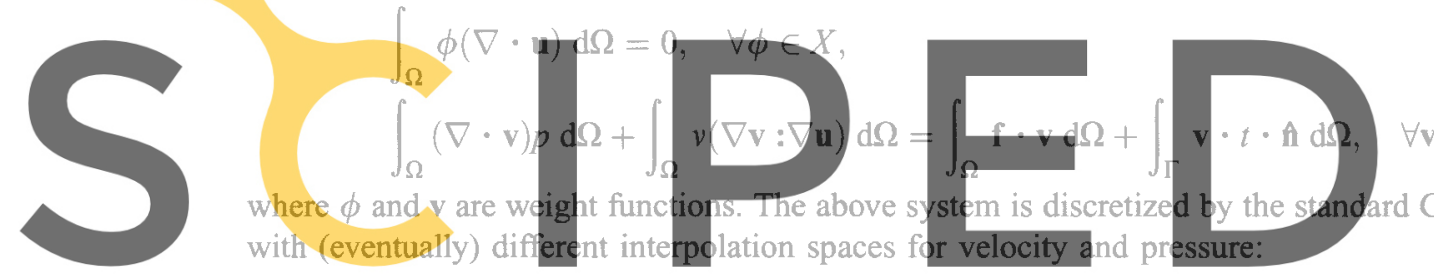

$$
p_{h}, \phi \in X_{h}=\operatorname{span}\left\{N_{p \mu}(\mathbf{x}), \mu=1, \ldots, N\right\},
$$

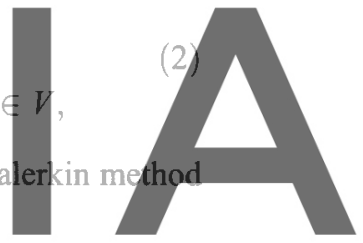

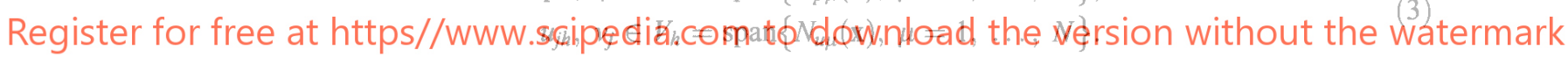

Note that since $\phi$ and $p$ have no derivatives in (2), elementwise discontinuous interpolations are allowed.

The discrete system obtained is

$$
\left[\begin{array}{cc}
0 & \mathbf{Q}^{\mathrm{T}} \\
-\mathbf{Q} & v \mathbf{K}
\end{array}\right]\left[\begin{array}{l}
\mathbf{P} \\
\mathbf{U}
\end{array}\right]=\left[\begin{array}{c}
\mathbf{0} \\
\mathbf{F}
\end{array}\right]
$$

where

$$
\begin{aligned}
& p_{h}=\sum_{\mu} p_{\mu} N_{p \mu}, \quad \mathbf{P}=\left[\begin{array}{c}
p_{1} \\
p_{2} \\
\vdots \\
p_{N}
\end{array}\right], \\
& u_{j h}=\sum_{\mu} u_{j \mu} N_{u \mu}, \quad \mathbf{U}=\left[\begin{array}{c}
\mathbf{u}_{1} \\
\mathbf{u}_{2} \\
\vdots \\
\mathbf{u}_{N}
\end{array}\right], \\
& Q_{\mu k v v}=\int N_{u \mu, k} N_{p v} \mathrm{~d} \Omega,
\end{aligned}
$$


To achieve a convergent approximation, the choice of the pair $\left\{X_{h}, V_{h}\right\}$ is crucial. It must satisfy the 'Brezzi-Babǔska' condition, otherwise oscillations in pressure are obtained. This condition is written as $^{4-6}$

$$
\inf _{q_{h} \in X_{h}-\{0\}} \sup _{\mathbf{v}_{h} \in V_{h}-\{0\}} \frac{\int_{\Omega} q_{h} \nabla \cdot \mathbf{v}_{h} \mathbf{d} \Omega}{\left(\int_{\Omega}\left|\nabla \mathbf{v}_{h}\right|^{2}\right)^{1 / 2}\left(\int_{\Omega} q_{h}^{2}\right)^{1 / 2}} \geq C \neq C(h) .
$$

\section{DISCRETE VERSION OF THE BREZZI-BABǓSKA CONDITION}

In terms of FEM matrices (6) is written as

$$
\inf _{\mathbf{q} \in R^{N}-\{0\}} \sup _{\mathbf{v} \in R^{3 N}-\{0\}} \frac{\mathbf{q}^{\mathrm{T}} \cdot \mathbf{Q v}}{\left(\mathbf{v}^{\mathrm{T}} \cdot \mathbf{K v}\right)^{1 / 2}\left(\mathbf{q}^{\mathrm{T}} \cdot \mathbf{M}_{p} \mathbf{q}\right)^{1 / 2}}=\overline{B B} \geq C \neq C(h),
$$

where $\mathbf{M}_{p}$ is a 'mass matrix' for the pressure functions:

$$
M_{p \mu \nu}=\int_{\Omega} N_{p \mu} N_{p v}
$$

This expression can be further simplified. First note that $\mathbf{K}$ and $\mathbf{M}_{p}$ are symmetric, positive definite matrices. Then, through the change of variables
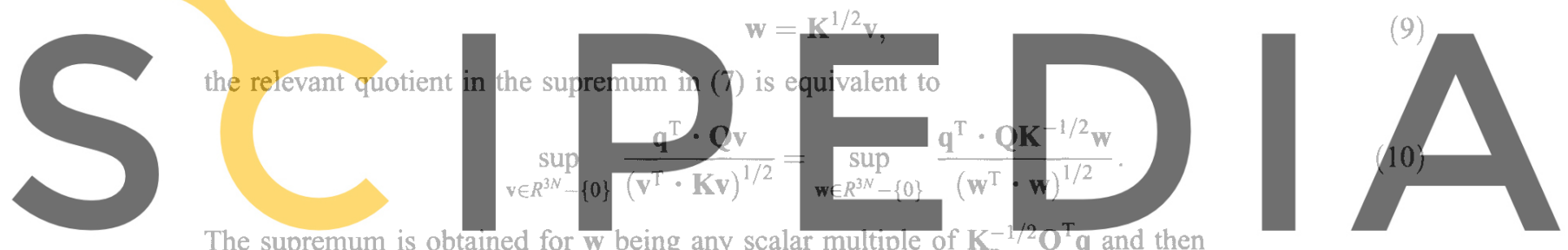

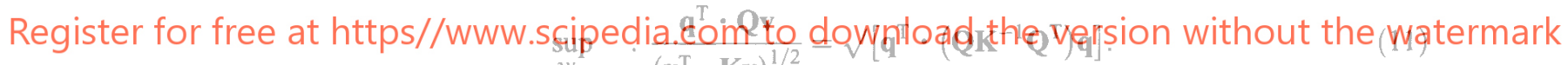

$$
\mathbf{v} \in R^{3 N}-\{0\} \quad\left(\mathbf{v}^{\mathrm{T}} \cdot \mathbf{K v}\right)^{1 / 2}
$$

Replacing in (7) and squaring gives

$$
\overrightarrow{B B}^{2}=\sup _{\mathbf{q} \in R^{N}-\{0\}} \frac{\mathbf{q}^{\mathrm{T}} \cdot\left(\mathbf{Q} \mathbf{K}^{-1} \mathbf{Q}^{\mathbf{T}}\right) \mathbf{q}}{\mathbf{q}^{\mathrm{T}} \cdot \mathbf{M}_{p} \mathbf{q}} .
$$

Again this expression is simplified by the auxiliary transformation $\mathbf{y}=\mathbf{M}^{1 / 2} \mathbf{q}$, obtaining

$$
\overline{B B}^{2}=\sup _{\mathbf{y} \in R^{N}-\{0\}} \frac{\mathbf{y}^{\mathrm{T}} \cdot\left(\mathbf{M}_{p}^{1 / 2} \mathbf{Q} \mathbf{K}^{-1} \mathbf{Q}^{\mathrm{T}} \mathbf{M}_{p}^{-1 / 2}\right) \mathbf{y}}{\mathbf{y}^{\mathrm{T}} \cdot \mathbf{y}} .
$$

However, this infimum is the minimum eigenvalue of the product matrix, i.e.

$$
\overline{B B}^{2}=\text { minimum eigenvalue of }\left\{\mathbf{M}_{p}^{-1 / 2} \mathbf{Q} \mathbf{K}^{-1} \mathbf{Q}^{\mathrm{T}} \mathbf{M}_{p}^{-1 / 2}\right\} \text {. }
$$

Finally we recall that eigenvalues are invariant under similarity transformations and we apply such a transformation with the matrix $\mathbf{M}_{p}^{1 / 2}$ :

$$
\overline{B B}^{2}=\text { minimum eigenvalue of }\left\{\mathbf{Q K} \mathbf{K}^{-1} \mathbf{Q}^{\mathrm{T}} \mathbf{M}_{p}^{-1}\right\} .
$$




\section{EQUIVALENCE TO THE PERIODIC PROBLEM}

Fourier analysis allows diagonal or block-diagonal decomposition of discrete periodic operators. ${ }^{7}$ Here we will use this technique to compute the complete set of eigenvalues of the matrix product involved in expression (15). As usual, the full power of Fourier analysis is attained in the case of linear operators with periodic boundary conditions. Here periodicity is assumed at both the continuum and discrete levels. For instance, consider the mesh in Figure 1. It is composed of $7 \times 7 \mathrm{Q} 2 / \mathrm{P} 1$ elements. To have a periodic problem at the continuum level, the boundary conditions should be periodic on opposing sides of the domain. On the other hand to have a periodic problem at the discrete level, the discretization at opposing sides should match perfectly as is the case for the mesh in the figure. In this way the discrete boundary conditions consist simply of identifying the corresponding degrees of freedom on the opposing sides. The same is true for the meshes in Figures 2 and 4. The first one is composed of $7 \times 7$ 'macroelements' divided into six Q1/P0 elements each (see Figure 3). The second one is composed of $3 \times 3$ macroelements divided into $3 \times 3$ Q1/P0 elements each (see Figure 5).

Referring to Figure 1, the total number of degrees of freedom is, once the degrees of freedom on opposing nodes have been eliminated through the periodic boundary conditions, $N_{\text {dof }}=m^{2} n_{\text {dof }}=7^{2} \times 11=847$, where $m$ is the number of identical macroelements repeated in each direction and $n_{\text {dof }}$ is the number of degrees of freedom per macroelement. The matrix product involved in the computation of the $\overline{B B}$ constant in (15) is of order $N_{\mathrm{dof}, p} \times N_{\mathrm{dof}, p}$, where $N_{\text {dof }, p}=m^{2} n_{\text {dof }, p}$ is the total number of pressure degrees of freedom and $n_{\text {dof }, p}=3$ is the number of degrees of freedom per macroelement. The power of Fourier analysis allows the decomposition of the

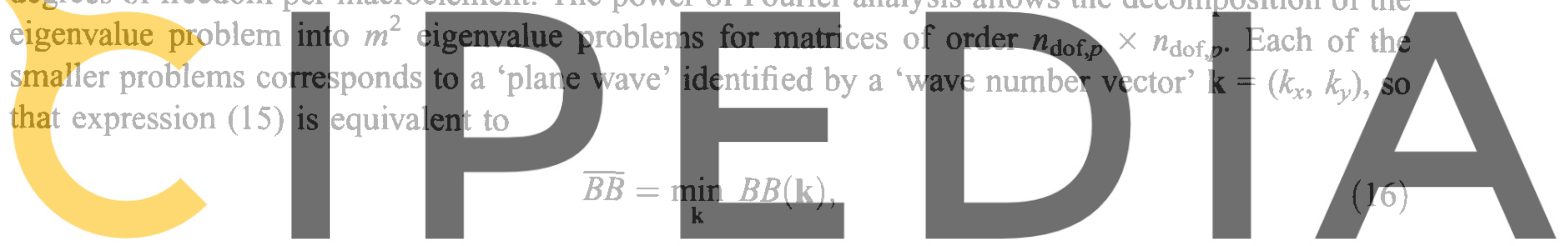

where $B B(\mathbf{k})$ is a Brezzi-Babǔska constant for the eigenspace spanned by the particular plane wave: Register for free at https//www.scipedia.com to download the version without the watermark $B B(\mathbf{k})^{2}=$ minimum eigenvalue of $\left\{\mathbf{Q}_{\mathbf{k}} \mathbf{K}_{\mathbf{k}}^{-1} \mathbf{Q}_{\mathbf{k}}^{\mathrm{T}} \mathbf{M}_{p \mathbf{k}}^{-1}\right\}$.

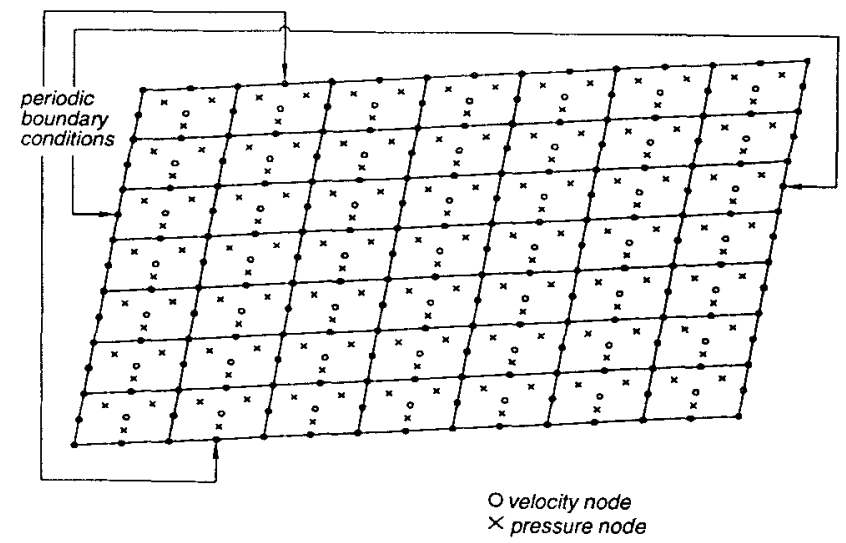

Figure 1 . FEM mesh composed of $7 \times 7 \mathrm{Q} / \mathrm{P} 0$ elements with periodic boundary conditions 


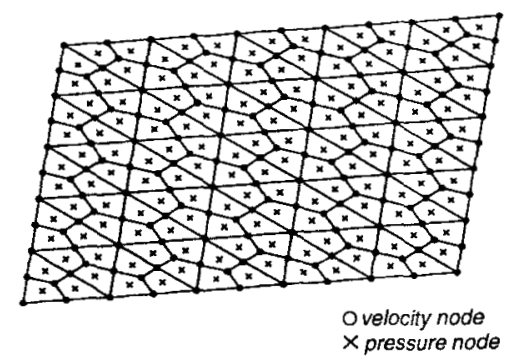

Figure 2. FEM mesh composed of $\mathrm{Q} 1 / \mathrm{P} 0$ macroelements with periodic boundary conditions

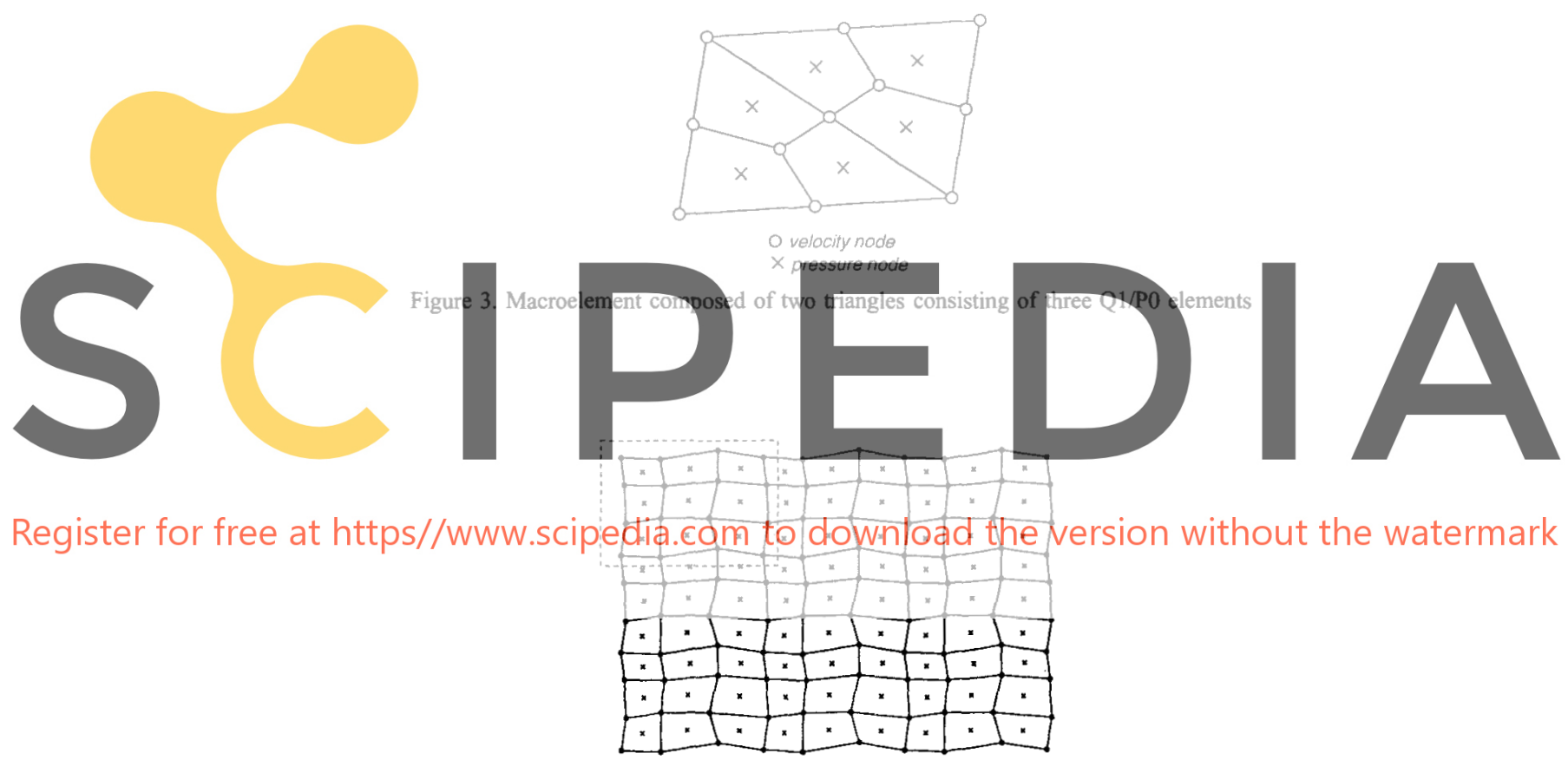

Figure 4. FEM mesh composed of $3 \times 3$ macroelements based on the Q1/P0 element. The macroelement is shown enclosed in a broken rectangle

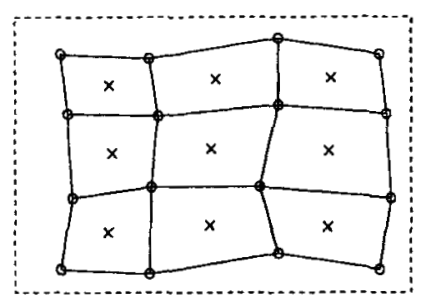

Figure 5. Basic macroelement of Figure 4 composed of $3 \times 3 \mathrm{Q} 1 / \mathrm{P} 0$ elements 
An equivalent criterion for stability is based on the determinant of the reduced system obtained for pressures when the momentum equation is solved for velocities and they are replaced in the continuity equation:

$$
\operatorname{det}\left(\mathbf{A}_{\mathbf{k}}\right)=\operatorname{det}\left\{\mathbf{Q}_{\mathbf{k}}^{\mathrm{T}} \mathbf{K}_{\mathbf{k}}^{-1} \mathbf{Q}_{\mathbf{k}}\right\}
$$

We now review the most known pairs of interpolation spaces.

\section{DISCONTINUOUS PRESSURE ELEMENTS}

QI/P0 (Figure 6). In the figure we see the results of the previous analysis applied to the Q1/P0 element, i.e. bilinear velocities and constant discontinuous pressures. In the upper part of the graph we see the $B B$ number from (17) and the second criterion (18) as functions of $k_{x}$ for several $k_{y}$ Also shown is a $3 \mathrm{D}$ elevation view of each criterion on the $\left(k_{x}, k_{y}\right)$ plane. As is usual in Fourier analysis, the analysis is restricted to a square of side $2 \pi$ in each direction. The points marked with circles in the corners of the square are unstable modes where both criteria vanish. Apparently there also exists an unstable mode at the origin $\mathbf{k}=0$, but this is the constant rigid mode $u, v, p=$ const. which has to be eliminated from the analysis when periodic boundary conditions are imposed. It could be thought at first sight that there are four unstable modes. In fact, owing to the periodicity of the transformed function, the nodes at the corner are all the same and must be counted as one-quarter each, while those modes at the sides must be counted as one-half each. In the lower part of the figure we see the aspect that the pressure takes for this unstable mode. Note that the two criteria coincide, i.e. they predict the
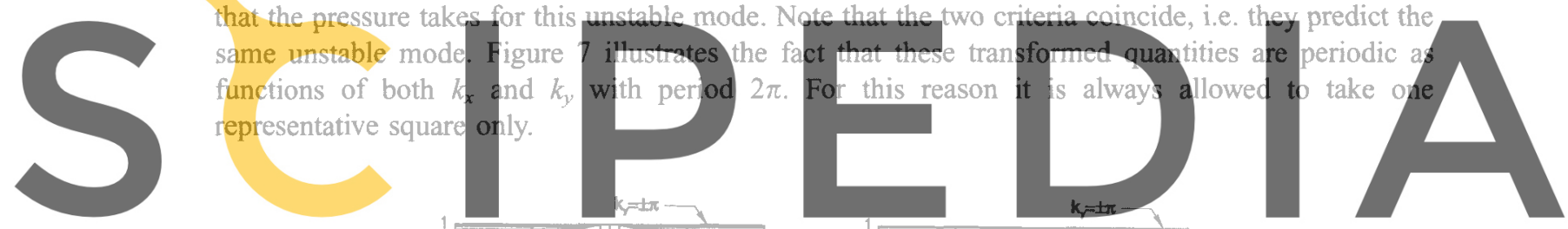

Register for free at https// $\overline{A H W W}$.scipedia.com to downtead the versien-without the watermark

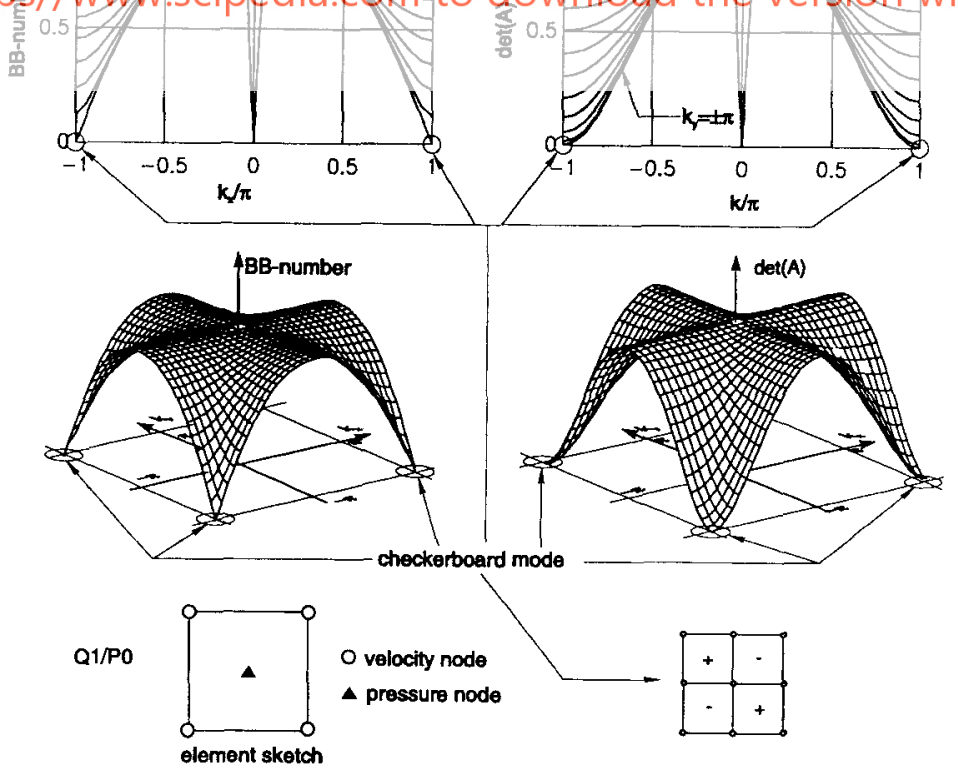

Figure 6. Stability analysis of the Q1/P0 element 


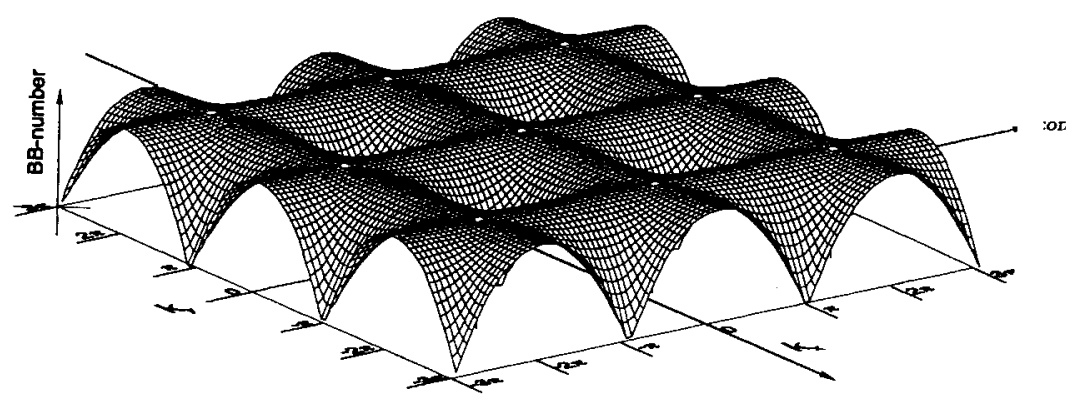

Figure 7. Periodicity of $B B\left(k_{x}, k_{y}\right)$ with respect to both $\kappa$ and $\lambda$

Q2/P1 (Figure 8). We see the plots corresponding to the Q2/P1 interpolation (biquadratic velocities and discontinuous elementwise linear pressures). Both criteria are strictly positive, since in all cases the apparently unstable mode at the origin is removed with the zero-mean-value restriction.

Q2/QI (Figure 9). If bilinear pressures are taken instead, the interpolation becomes unstable. Note that, in contrast with the Q1/P0 and Q2/P1 elements, the null eigenvalue at $\mathbf{k}=0$ is continuously reached from $\mathbf{k} \neq 0$. The fact that the unstable mode is at the origin $\mathbf{k}=0$ does not imply that it is a smooth 'low-frequency' mode. The corresponding variations in pressure are sketched in the lower part of the figure. The high-frequency variation is due to variations in the pressure inside the 'base molecule" (i.e. the set of degrees of freedom associated with each period), which has four pressure nodes. This kind of mode form is called ax 'optical' branch in crystal physics.

Q2/Q1 (Figure 10). If in addition, the intemal node of the Lagranglan biquadra velocities is eliminated (eight-velocity-node element, also called the "serendipity" the instability becomes complete (nfinite in the limit) sot of modes. In this case all the modes are unstable.

Q2/P1 (Figure II). Now, coming back to the discontinuous linear interpolation for pressures,

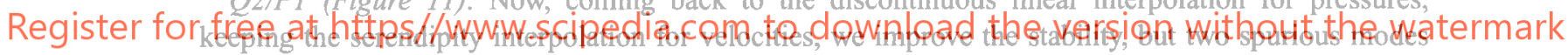
still remain: $\mathbf{k}=\{ \pm \pi, 0\},\{0, \pm \pi\}$.

Q2/P0 (Figure 12). The interpolation is fully stabilized if the pressure space is reduced to constant pressures by element only. However, as is well known, this causes a loss of accuracy of one-half order.

Pl(disc)/PO (Figure 13). We pass to review the triangular elements. In this case linear discontinuous (non-conforming) velocities are taken. The velocity nodes are taken at the mid-side of the elements. This happens to be nicely stable.
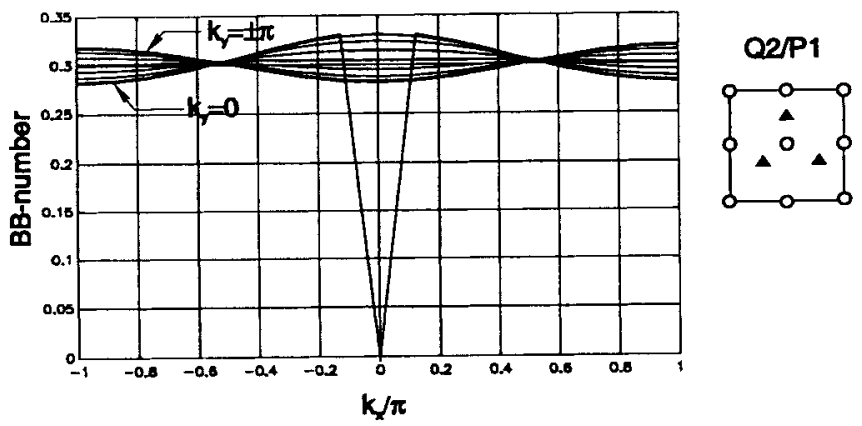

Figure 8. Stability analysis of the $\mathrm{Q} 2 / \mathrm{P} 1$ element 

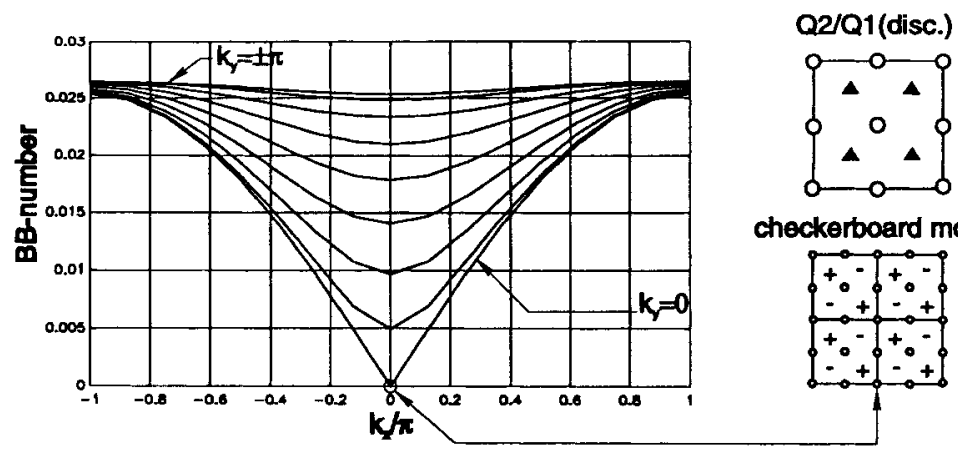

Figure 9. Stability analysis of the Q2/Q1 element

checkerboard mode

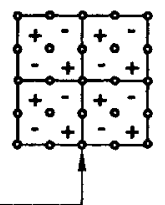

Q2/Q1
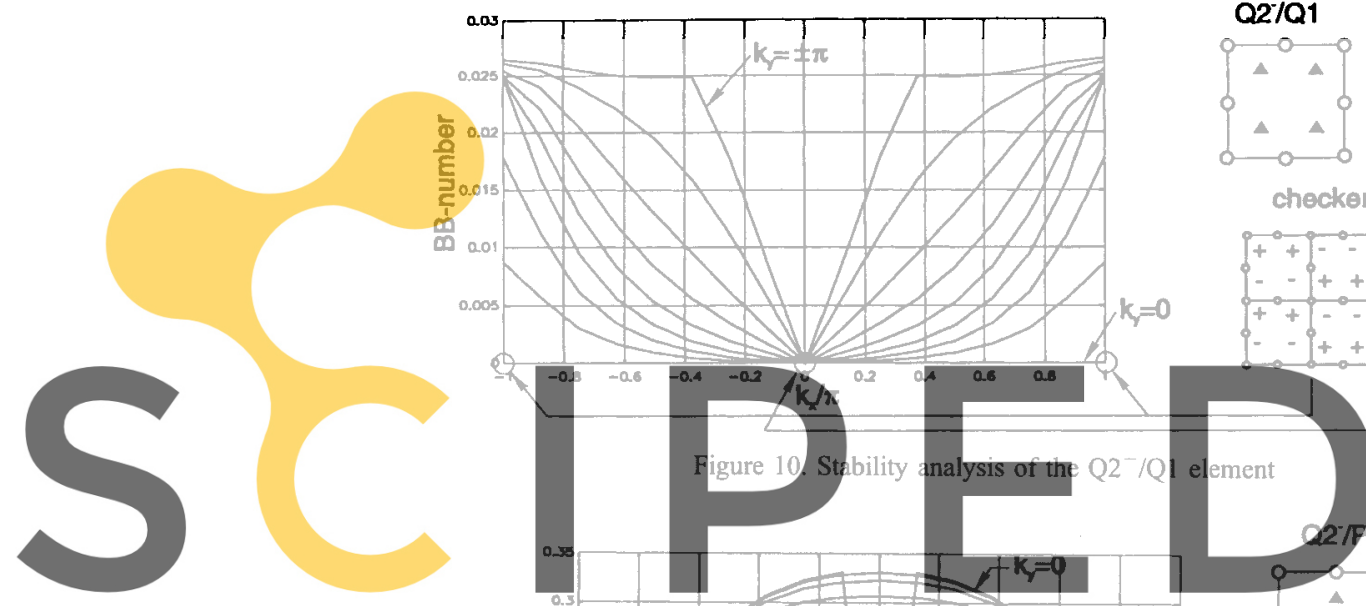

checkerboard modes

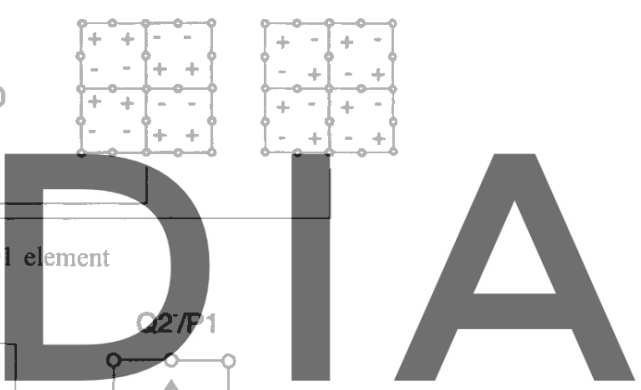

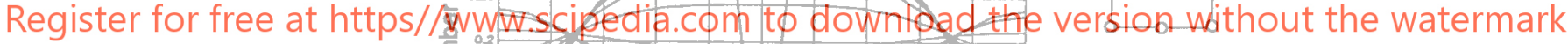

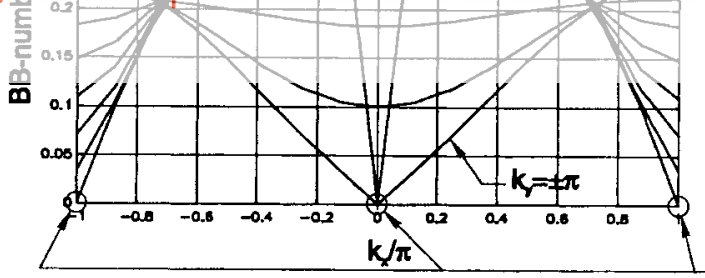

checkerboand modes

Figure 11. Stability analysis of the Q2 $/ \mathrm{P} 1$ element

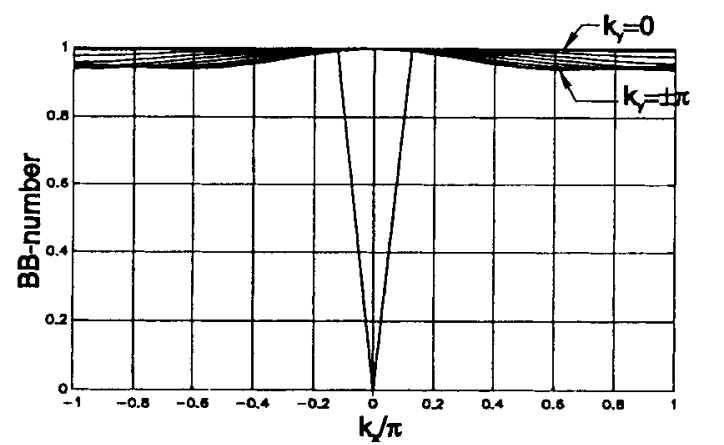

Q2 $/ P 0$<smiles>C1OOOOOOOO1</smiles>

Figure 12. Stability analysis of the $\mathrm{Q} 2^{-} / \mathrm{P} 0$ element 


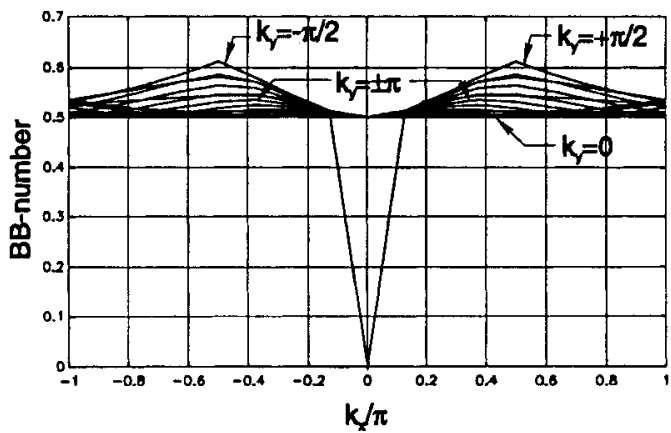

\section{P1(non conf.)/PO}

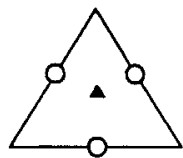

Figure 13. Stability analysis of the P1(non-conforming)/P0 element

P2/PI (Figure 14). This is the triangular version of the Q2/P1 element, but in contrast with it, it is unstable with two spurious modes at $\mathbf{k}=\{0,0\}$.

$P 2 / P 0$ (Figure 15). The quadratic interpolation for velocities can be stabilized if the space of pressures is reduced to constant ones, but as for the Q2/P0 element, this does not have optimal convergence rates.

$P 2^{+} / P 1$ (Figure 16). The P2/P1 elements can be stabilized through the addition of a "bubble function' to the velocity space.
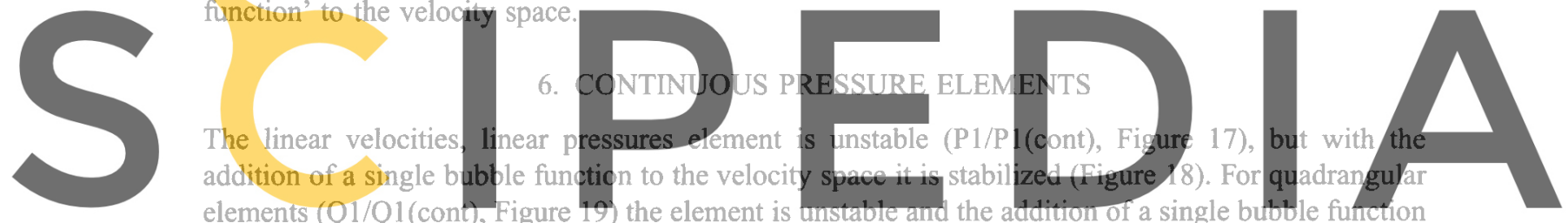

elements (Q1/Q1 (cont), Figure 19) the element is unstable and the addition of a single bubtle function does not suffice to stabilize it (Q1 ${ }^{+} / \mathrm{Q} 1$ (cont), Figure 20). The bubble function is, in master element co-

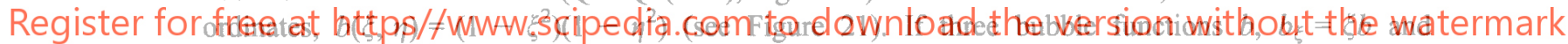
$b_{\eta}=\eta b$ (see Figures 22 and 23 ) are added instead $\left(\mathrm{Q} 1^{+3} / \mathrm{Q} 1\right.$ (cont), Figure 24), then the interpolation is stable.

On the other hand, second-order interpolation for velocities and first-order interpolation for pressures give combinations which are generally stable, as is the case for P2/P1(cont) (Figure 25), Q2/ Q1(cont) (Figure 26) and Q2 ${ }^{-} / \mathrm{Q} 1$ (cont) (Figure 27).
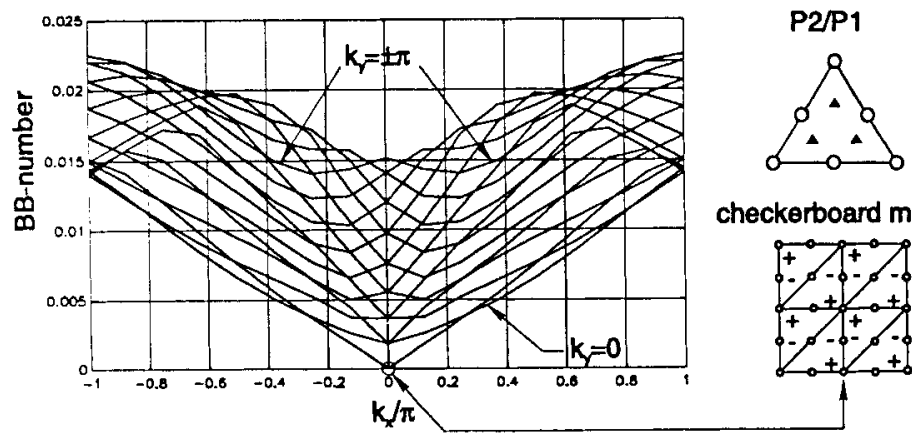

checkerboard modes

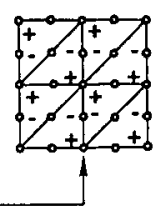

Figure 14 . Stability analysis of the $\mathrm{P} 2 / \mathrm{P} 1$ element 


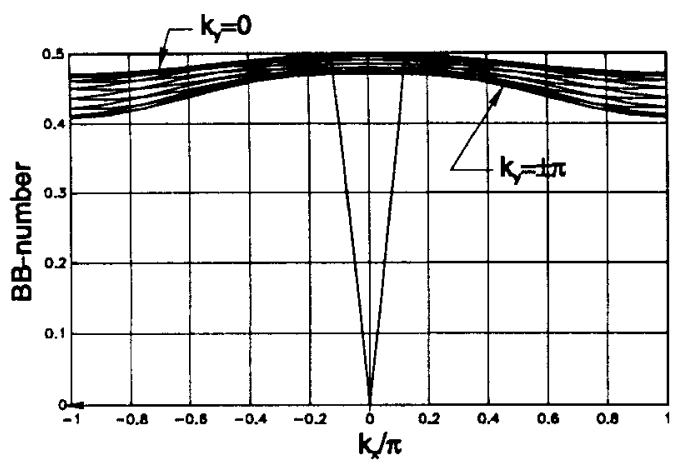

P2/PO

Figure 15. Stability analysis of the $\mathrm{P} 2 / \mathrm{P} 0$ element
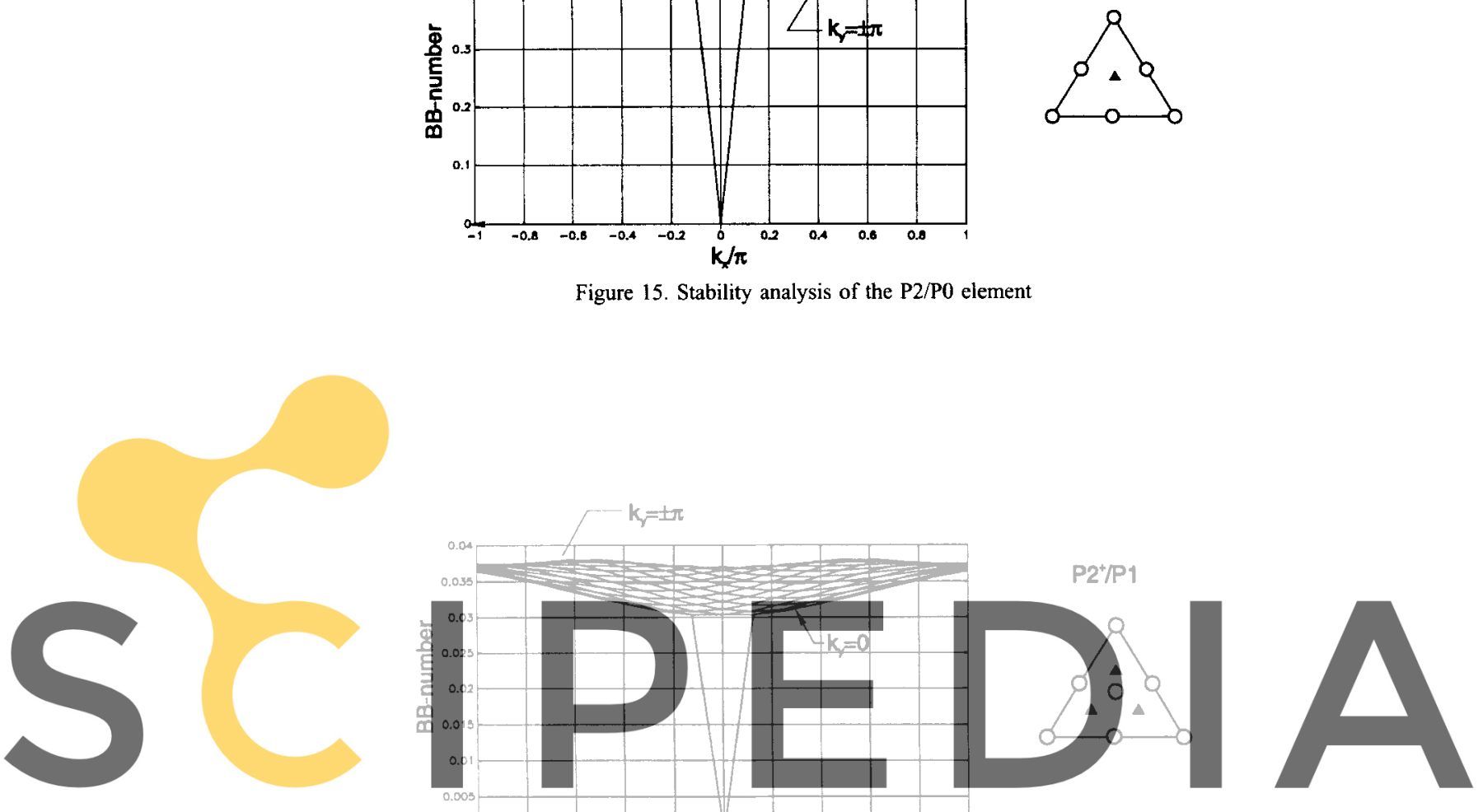

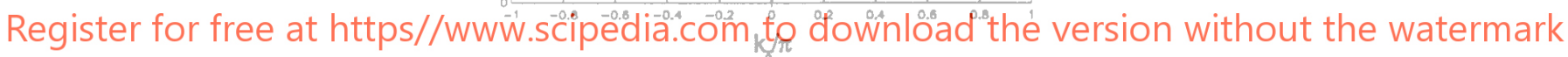

Figure 16. Stability analysis of the $\mathrm{P} 2^{+} / \mathrm{P} 1$ element

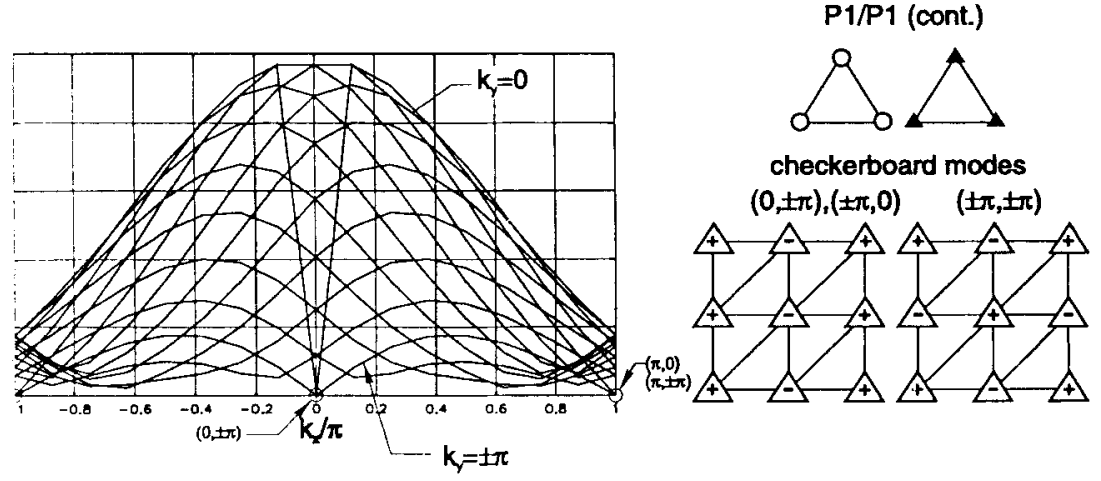

Figure 17 . Stability analysis of the $\mathrm{P} 1 / \mathrm{P} 1$ (cont) element 


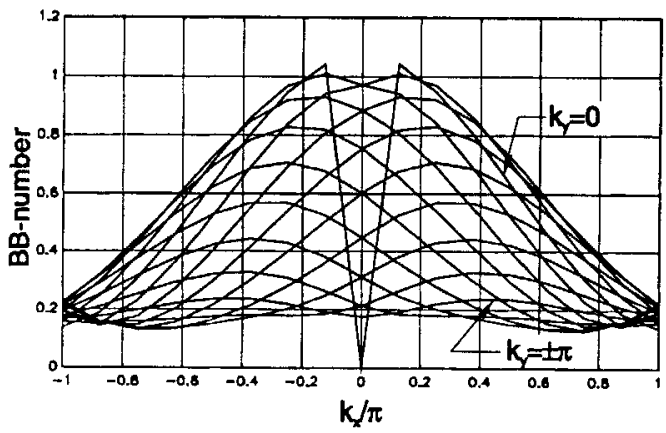

\section{P1+/P1 (cont.)}
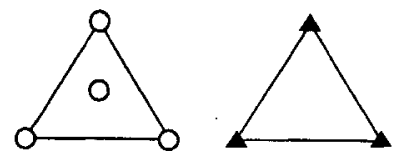

Figure 18 . Stability analysis of the $\mathrm{P} 1^{+} / \mathrm{P} 1$ (cont) element

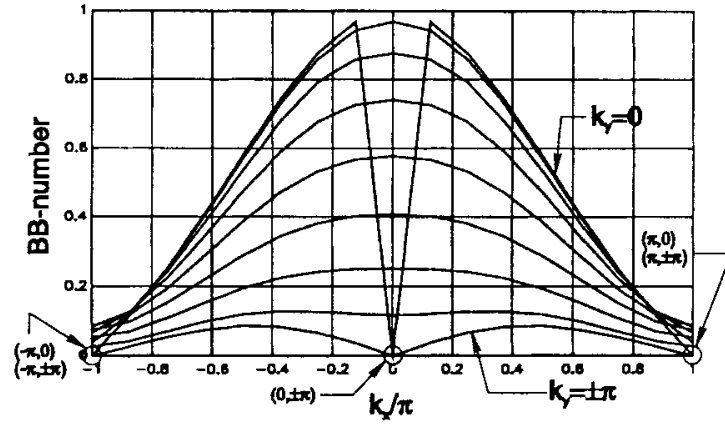

Q1/Q1 (cont.)

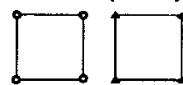

checkerboard modes $(0, \pm \pi),( \pm \pi, 0)( \pm \pi,+\pi)$

Figure 19. Stability analysis of the Q1/Q1(cont) element

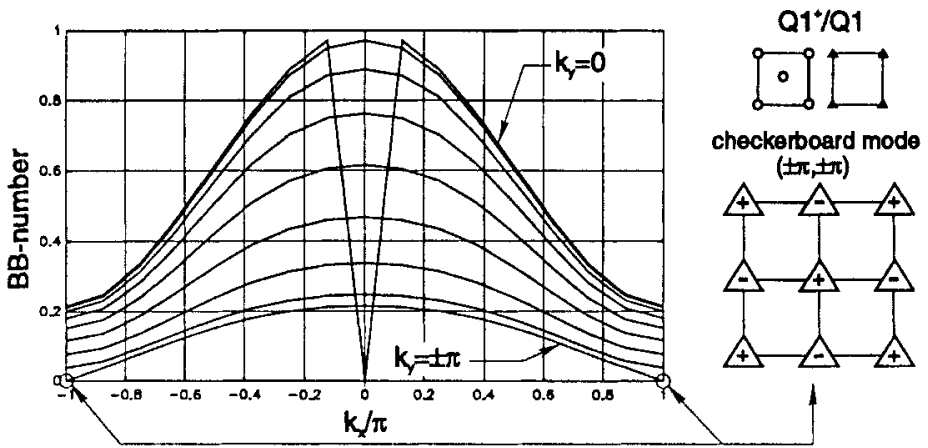

Figure 20. Stability analysis of the $\mathrm{Q}^{+} / \mathrm{Ql}$ (cont) element

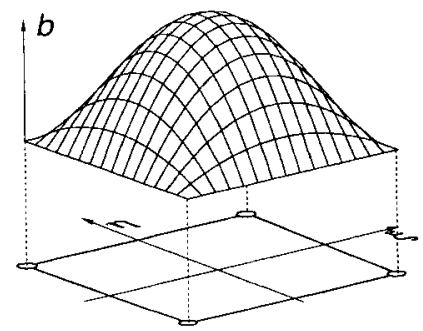

Figure 21. Bubble function $b(\xi, \eta)$ for quadrangular elements 


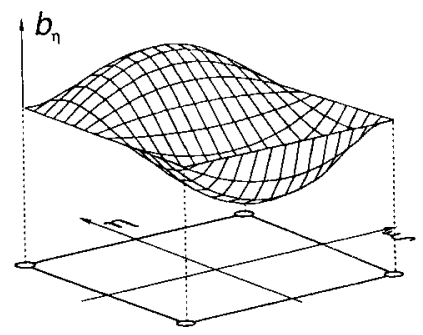

Figure 22. Bubble function $\xi b(\xi, \eta)$

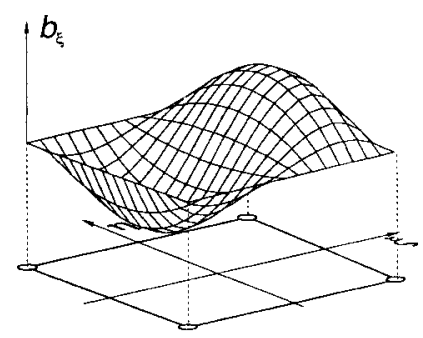

Figure 23. Bubble function $\eta b(\xi, \eta)$

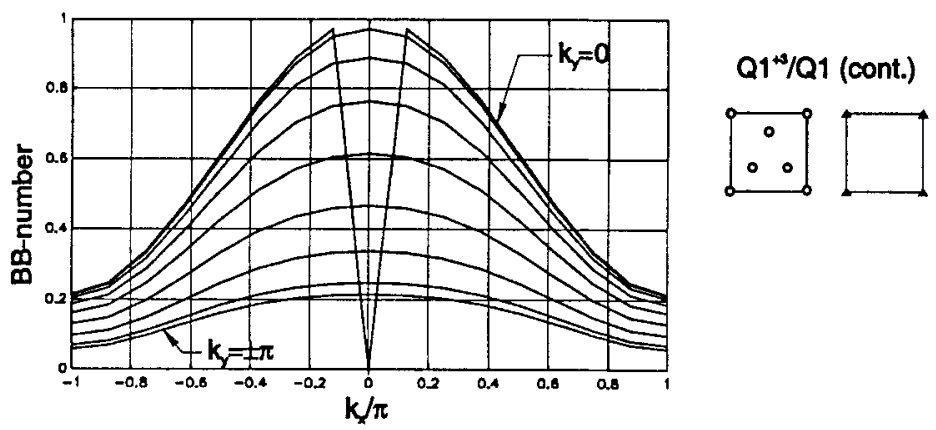

Figure 24. Stability analysis of the $\mathrm{Q} 1^{+3} / \mathrm{Q} 1$ (cont) element

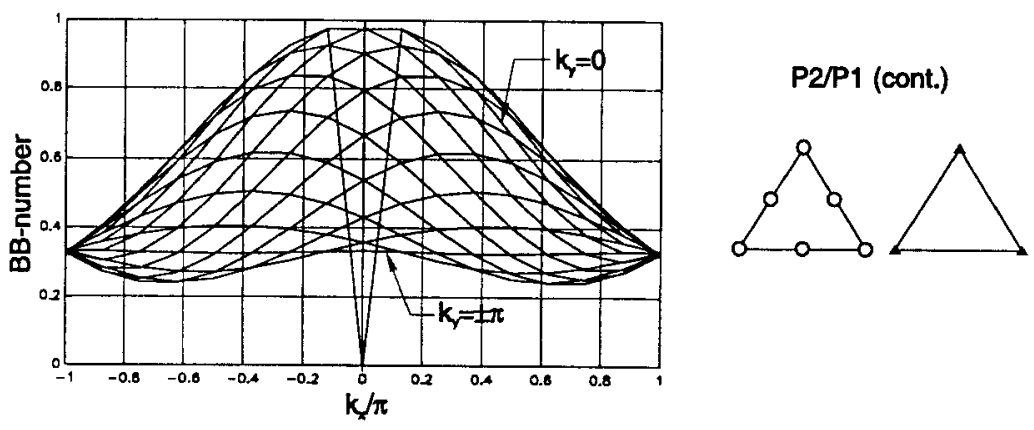

Figure 25. Stability analysis of the P2/P1(cont) element 

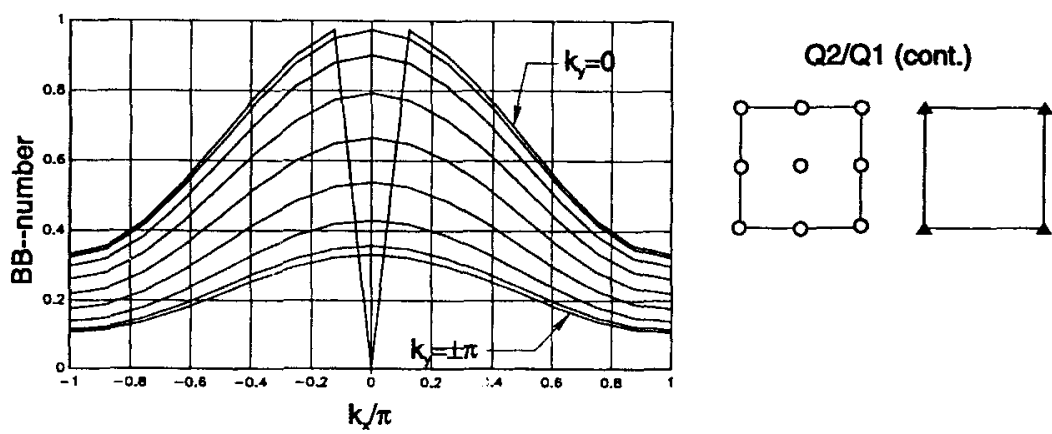

Figure 26. Stability analysis of the Q2/Q1(cont) element

\section{STABILIZED METHODS ${ }^{8,9}$}

Pairs of interpolation spaces satisfying the $B B$ condition are difficult to obtain. An alternative is the stabilization of a priori unstable approximations through addition of mesh-size-dependent stabilization terms. These terms are all very similar, but the arguments involved differ from one author to another. For instance, the 'velocity correction methods' of Schneider et al. ${ }^{10}$ and Kawahara and Ohmiya ${ }^{11}$ propose a stabilized algorithm based on operator splitting. The scheme of Zienkiewicz et al. ${ }^{12}$ is also based on operator splitting. Other schemes, e.g. those of Douglas and Wang, ${ }^{13}$ Hughes et al. ${ }^{14}$ and Tezduyar et al., ${ }^{15}$ are based on Petrov-Galerkin techniques such as SUPG (streamline upwind PetrovGalerkin), PSPG (pressure-stabilizing Petrov-Galerkin) or GLS (Galerkin least squares).

The main disadvantage of stabilized methods is the addition of 'free parameters' (the 'amount' of stabilization to be added), in contrast with the stable formulations which are parameter-free. In fact, the two approaches are not so different: it can be shown that (under certain restrictions) stabilization terms are equivalent to the enlargement of the velocity space by the addition of certain (not explicitly known) 'virtual' bubble functions. ${ }^{16}$

\section{A STABILIZED ALGORITHM BASED ON THE EXTENSION OF SUPG TO SYSTEMS}

We recall the stabilized method presented in Reference 8 . It is based on a straightforward extension of the basic SUPG scheme to systems of equations in such a way as to keep the well-known phenomenon of 'superconvergence', i.e. exact modal values are obtained in some restricted class of problems (1D, uniform mesh, no source term).

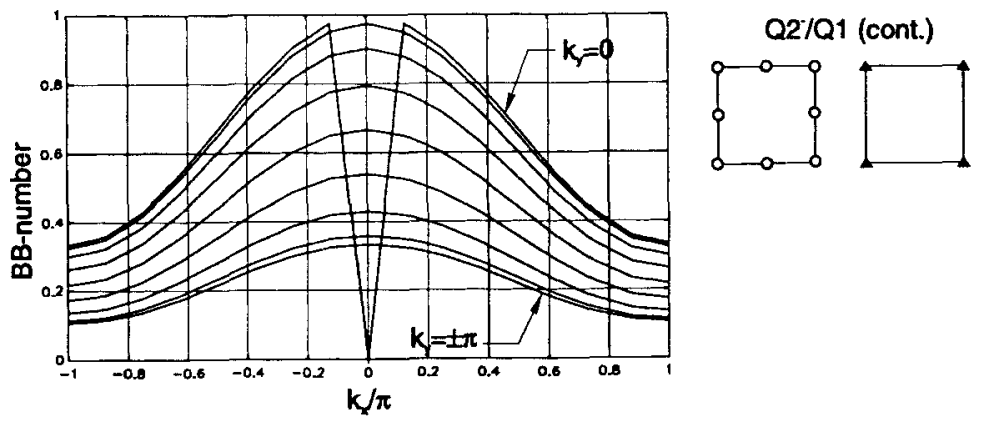

Figure 27. Stability analysis of the $\mathrm{Q} 2^{-} / \mathrm{Q} 1$ (cont) element 
For the 1D scalar advective-diffusive equation with constant coefficients,

$$
a u_{, x}=k u_{, x x},
$$

superconvergence is obtained with the discrete system

$$
a \frac{u_{i+1}-u_{i-1}}{2 \Delta x}=\left(k+k^{\text {num }}\right) \frac{u_{i+1}-2 u_{i}+u_{i-1}}{\Delta x^{2}},
$$

with

$$
k^{\mathrm{num}}=\frac{a \Delta x}{2} \psi(P e), \quad \frac{P e=u \Delta x}{k}, \quad \psi(P e)=\frac{1}{\tanh (P e)}-\frac{2}{P e},
$$

where $\psi$ is called a 'magic function' (see Figure 28). The latter can be replaced by other alternatives which give the same convergence rates, but superconvergence is obtained only if the definition is taken as in (21). This discrete system can be cast in a weighted residual form if the following weighting functions $W_{i}$ are used:

$$
W_{i}=N_{i}+P_{i}, \quad P_{i}=\tau a N_{i, x}, \quad \tau=\frac{\Delta x}{2|a|} \psi(P e) .
$$

$P_{i}$ are the perturbations to the standard Galerkin formulation to add the necessary numerical diffusion. The intrinsic time $\tau$ can be rewritten as

$$
\tau=\frac{\Delta x^{2}}{2|a|} \phi(P e), \quad \phi(P e)=\frac{\psi(P e)}{P e},
$$

where $\phi$ is a magic function. The latter is bounded and well-behaved, as can be seen from the plot in Figure 28 .

For advective-diffusive systems of equations

$$
\mathbf{A} \mathbf{U}_{, x}=\mathbf{K} \mathbf{U}_{, x x}, \quad \mathbf{U} \in R^{m}, \quad \mathbf{A}, \mathbf{K} \in R^{m \times m},
$$

the expression for the scalar intrinsic time is extended to a matrix of intrinsic time scales

$$
t=\frac{\Delta x^{2}}{2} \phi\left(\mathbf{K}^{-1} \mathbf{A} \Delta x\right) \mathbf{K}^{-1} \text {. }
$$

As usual, $\phi$ is evaluated on its matrix argument through an eigenvalue decomposition. Expression (24) trivially reduces to (22) for the scalar case. This extension of SUPG to systems preserves the phenomenon of superconvergence, i.e. exact nodal values are obtained for problems with homogeneously spaced grids and no source term. The extension to multidimensional systems

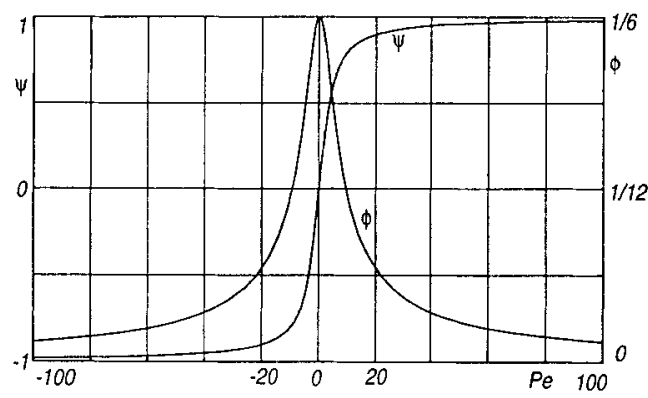

Figure 28. 'Magic functions' $\phi$ and $\psi$ 
amounts to computing an equivalent one-dimensional matrix of intrinsic times scales $\tau_{x}$ as in (24) but using the projected jacobians $\mathbf{A}_{x}$ and $\mathbf{K}_{x x}$, and similarly for the $y$ - and $z$-directions. The global matrix is computed as

$$
\tau^{-1}=\tau_{x}^{-1}+\tau_{y}^{-1}+\tau_{z}^{-1} .
$$

The multidimensional version of the Stokes system (1) can be cast as an advective-diffusive one (23) with the definitions

$$
\mathbf{U}=\left[\begin{array}{c}
\mathbf{u} \\
p
\end{array}\right], \quad \mathbf{A}_{j} k_{j}=\left[\begin{array}{cc}
\mathbf{0} & \mathbf{k} \\
\mathbf{k}^{\mathrm{T}} & 0
\end{array}\right], \quad \mathbf{K}_{j l}=\delta_{j l}\left[\begin{array}{cc}
\nu \mathbf{I} & 0 \\
0 & \varepsilon \delta_{j l}
\end{array}\right] .
$$

Here $\mathbf{k}$ is an arbitrary wave number vector and $\varepsilon$ has been added to make the matrices $\mathbf{K}_{j l}$ non-singular. This property is needed to compute their inverse in expression (24). However, this inconsistency is added only for the computation of the matrix of intrinsic time scales. Once this is calculated and then the weighting functions, they are applied to the unmodified (without the $\varepsilon$-term) Stokes system. Applying expressions (24) and (25), the following matrix of intrinsic time scales is obtained:

$$
\tau=\operatorname{diag}\{\alpha, \alpha, \alpha, \beta\}, \quad \alpha=\frac{\sqrt{ } \varepsilon h^{2} \xi}{2 h \sqrt{ } \nu+24 v \sqrt{ } \varepsilon \xi}, \quad \beta=\frac{h \xi}{6} \sqrt{\left(\frac{v}{\varepsilon}\right)},
$$

where $\xi=\psi(h / \sqrt{ }(\varepsilon v))$ and $\operatorname{diag}\{a, b, c \ldots\}$ stands for a diagonal matrix with diagonal entries $a, b, c \ldots$. The numerical diffusion matrix is

$$
\mathbf{K}_{i j}^{\mathrm{num}} k_{i} k_{j}=\left[\begin{array}{cc}
\beta \mathbf{k k}^{\mathrm{T}} & 0 \\
0 & \alpha k^{2}
\end{array}\right]
$$

for an arbitrary $\mathbf{k} \in \mathbb{R}^{3}$ and the discretized system is

$$
\left[\begin{array}{cc}
v \mathbf{K}+\beta \mathbf{G} & \mathbf{Q} \\
-\mathbf{Q}^{\mathrm{T}} & \alpha \mathbf{H}
\end{array}\right]\left[\begin{array}{l}
\mathbf{u} \\
\mathbf{P}
\end{array}\right]=\left[\begin{array}{c}
\mathbf{F} \\
\alpha \mathbf{S}
\end{array}\right]
$$

where the standard finite element matrices and interpolations are assumed:

$$
\begin{aligned}
H_{\mu, v} & =\int_{\Omega} N_{p \mu, k} N_{p v, k} \mathrm{~d} \Omega, & G_{i \mu, j v} & =\int_{\Omega} N_{u \mu, i} N_{u v, j} \mathrm{~d} \Omega, \\
F_{i \mu} & =\int_{\Omega} f_{i} N_{u \mu} \mathrm{d} \Omega, & S_{\mu} & =\int_{\Omega} f_{i} N_{p \mu, i} \mathrm{~d} \Omega .
\end{aligned}
$$

System (29) corresponds to the centred Galerkin discretization of the PDE system

$$
-v \Delta \mathbf{u}-\beta \nabla(\nabla \cdot \mathbf{u})+\nabla p=\mathbf{f}, \quad \nabla \cdot \mathbf{u}-\alpha \Delta p=-\alpha(\nabla \cdot \mathbf{f})
$$

and can be obtained from the original Stokes system by adding $-\beta$ times the gradient of the continuity equation to the momentum equation and $-\alpha$ times the divergence of the momentum equation to the continuity equation. Since the systems are equivalent, the resulting stabilized scheme has the same precision (in the sense of order of truncation error) independently of $\alpha$ and $\beta$.

\section{FOURIER ANALYSIS FOR STABILIZED SCHEMES}

Recently a great deal of research work ${ }^{12,14,17}$ has been carried out to circumvent the Brezzi-Babǔska restriction with equal-order schemes. It has been shown ${ }^{9}$ that all these formulations are based on the introduction (with different justifications varying from one author to another) of a stabilizing term proportional to the discrete version of $\Delta p$ in the continuity equation, like the term $\alpha \mathbf{H}$ in our 
formulation. Furthermore, the formulation of Frey et al. ${ }^{17}$ is the closest to ours, since it introduces a term equivalent to a discrete $\nabla(\nabla \cdot \mathbf{u})$ in the momentum equation, like the term $\beta \mathbf{G}$ in ours. This term represents physically a bulk viscosity term. This term can also be introduced by a straightforward application of the Taylor-Galerkin method. ${ }^{9}$

Most of the stabilized methods are basically of the form (29). The case $\alpha=\beta=0$ corresponds to the Galerkin non-stabilized case. The case $\beta=0, \alpha=\alpha^{\prime} h^{2} / 2 v$ corresponds to the stabilized method of Hughes et al. ${ }^{14}$ The scheme of Frey et al. ${ }^{17}$ (in the Stokes regime and for linear elements) is obtained with $\alpha=2 m h^{2} / v$ and $\beta=\lambda m u^{2} h^{2} / 4 v$, where $\lambda$ and $m$ are $O(1)$ constants defined in that work.

A stability analysis has been performed on the P1/P1 and Q1/Q1 elements, but using the $\operatorname{det}(\mathbf{A})$ criterion (18). The usual criterion is no longer valid owing to the presence of the stabilizing term. The criteria for the Galerkin $(\alpha=\beta=0)$ and stabilized $(\beta=0, \alpha=0 \cdot 1)$ cases are shown in Figures 29 and 30 . Both criteria become strictly positive for all wave numbers with the stabilized formulation, whereas the Galerkin formulation has three checker-board modes for both elements.

A deeper analysis is needed to precisely determine the amount of stabilization needed to have a good compromise between stability and accuracy. We will concentrate on the mid-side spurious modes of the form $\{ \pm \pi, 00\}$, since they are purely one-dimensional (all quantities are constant along the $y$ - and $z$ directions), and a one-dimensional analysis is performed.

The 1D discrete system resulting from considering variations along the $x$-direction only is

$$
\begin{aligned}
-\alpha \frac{p_{i+1}-2 p_{i}+p_{i-1}}{h^{2}}+\frac{u_{i+1}-u_{i-1}}{2 h} & =-\alpha \frac{f_{i+1}-f_{i-1}}{2 h}, \\
\frac{p_{i+1}-p_{i-1}}{2 h}-(\beta+v) \frac{u_{i+1}-2 u_{i}+u_{i-1}}{h^{2}} & =\frac{f_{i+1}+4 f_{i}+f_{i-1}}{6},
\end{aligned}
$$

where $i$ is the node index running along the $x$-direction. Applying the Fourier discrete transform, the response function for pressure is obtained as

$$
G_{p, f}=\frac{\hat{p}}{\hat{f}}=h \frac{-i \sin (k h)\left\{(1 / 3)[2+\cos (k h)]+(\gamma / 4) \sin ^{2}(k h / 2)\right\}}{\gamma \sin ^{4}(k h / 2)+\sin ^{2}(k h)},
$$

where $k$ stands for $k_{x}$ and

$$
\gamma=\frac{16 \alpha(v+\beta)}{h^{2}}=\frac{16 \alpha v}{h^{2}}+\frac{16 \alpha \beta}{h^{2}}=\gamma_{\mathrm{I}}+\gamma_{\mathrm{II}}
$$

is a global stability parameter.

In Figure 31 we can see the effect of $\gamma$ on the response function. For $\gamma>0$ the singularity is removed at $k=\pi$. However, for too small $\gamma(\gamma \ll 1)$ an undesirable peak in the response function occurs near $k=\pi$. It is seen that for $\gamma \approx 1$ the peak is completely removed and a monotone response

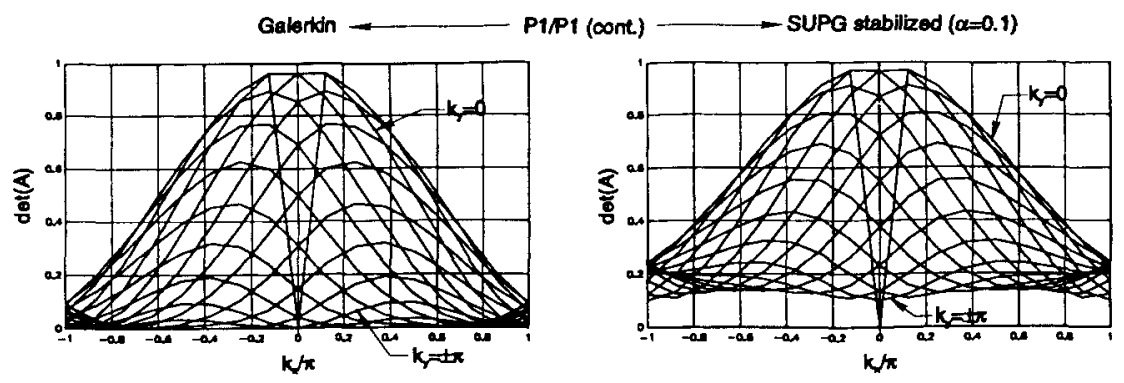

Figure 29. Analysis of the stabilized P1/P1 (cont) element 


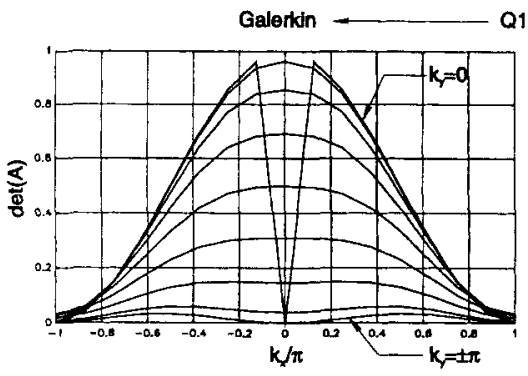

Q1/Q1 (cont.) — _ SUPG stabilized $(\alpha=0.1)$

Figure 30. Analysis of the stabilized Q1/Q1 (cont) element

curve is obtained. Numerical results from Reference 14 show that for the lid-drive cavity flow benchmark with a global stability parameter $\gamma$ ranging from 0.8 to 8 , oscillations are absent, whereas for $\gamma<0.08$, oscillations exist. These results are in perfect agreement with the previous discrete analysis.

As regards the proposed method, the global stability parameter is

$$
\gamma=\frac{4}{3} \xi \frac{\xi+6 \sqrt{\varepsilon}}{1+12 \sqrt{\varepsilon} \xi}
$$

where

$$
\tilde{\varepsilon}=\varepsilon v / h^{2}, \quad \xi=\psi(1 / \sqrt{\tilde{\varepsilon}}),
$$

$\tilde{\epsilon}$ being a non-dimensional version of $\varepsilon$. The relationship between $\gamma$ and $\tilde{\epsilon}$ is depicted in Figure 32 together with each of the terms in (34). We can see that irrespective of the choice of $\tilde{\epsilon}$ the algorithm is stable $(\gamma>0 \cdot 4)$. Note that for very small $\tilde{\epsilon}$ the first term $\gamma_{I}$ in (34) is not enough to stabilize, but the second term $\gamma_{I I}$ is. Thus in this case the bulk viscosity stabilization term $\beta \nabla(\nabla \cdot \mathbf{u})$ is indispensable.

With respect to accuracy, a careful analysis shows that a boundary condition on pressure has to be added, since the second-order term $\Delta p$ has been added. For instance $u, v=0$ ('solid wall') is an admissible boundary condition for the continuum problem, whereas for system (31) a boundary condition in pressure, e.g. $\partial p / \partial n=0$, has to be added. This last condition is artificial and distorts the numerical results near the boundaries. Consider, for instance, the 1D version of (31):

$$
-\alpha p^{\prime \prime}+u^{\prime}=-\alpha f^{\prime}, \quad p^{\prime}-(\beta+v) u^{\prime \prime}=f .
$$

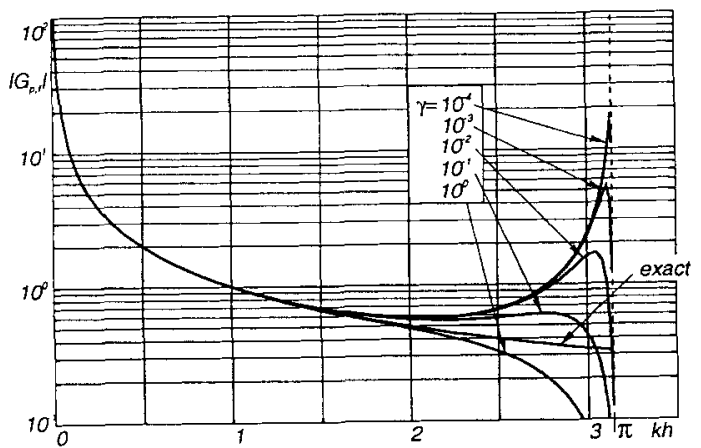

Figure 31. Response curves for a stability parameter ranging from $\gamma=10^{-4}$ to 1 


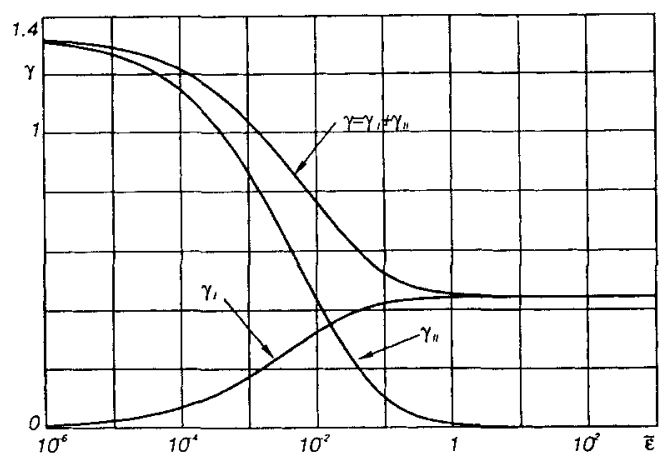

Figure 32. Stability parameter for the proposed method as function of 'free parameter' $\bar{\epsilon}$

If we take the derivative of the first equation and substitute for $u^{\prime \prime}$ from the second one, then a single equation for $p$ is obtained:

$$
-\alpha(\beta+v) p+p=f-\alpha(\beta+v) f .
$$

The roots of the characteristic equation are 0 and $\pm 1 /[\alpha(\beta+v)]$. The last two roots represent a boundary layer of thickness $\sqrt{ }[\alpha(\beta \mp \nu)]$. Then there is a compromise between stability (high $\alpha, \beta)$ and precision (low $\alpha, \beta)$. The choice $\alpha=O\left(h^{2} / v\right), \beta=O(v)$ is optimal: stability is within safe limits and the width of the 'pressure boundary layer' is of the order of roughly one element. ${ }^{8}$ This optimal choice is automatically captured in the present scheme for the whole range of $\tilde{\epsilon}$.

\section{A NUMERICAL EXAMPLE}

The well-known test of lid-driven cavity flow (see Figure 33) at $R e=1000$ is used to show the performance of the method. In Figure 34 we can see the velocity pattern on a mesh composed of $20 \times 20 \mathrm{Q} 1 / \mathrm{Q} 1$ elements with continuous interpolation for pressure and the stabilized method described here with $\tilde{\epsilon}=1$.

\section{CONCLUSIONS}

The criterion described here is based on Fourier analysis and allows an algebraic determination of the stability of a given pair of interpolation spaces. The test has been applied to the most known interpolations, giving the expected results. A deeper study is performed on stabilized algorithms to

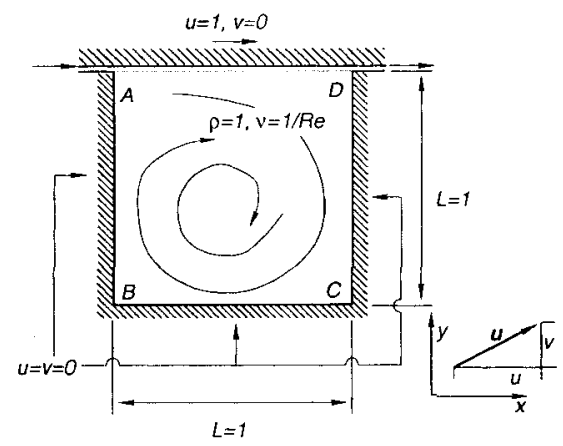

Figure 33. Lid-driven cavity flow: problem description and geometry 


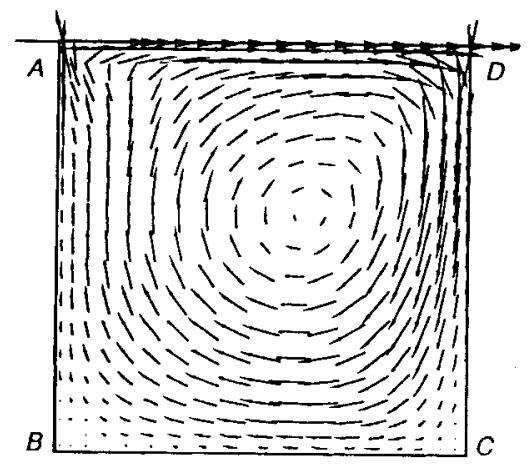

Figure 34. Lid-driven cavity flow: velocity vectors at $R e=1000$

determine the amount of numerical additive that is needed to achieve a stable approximation without significant loss of accuracy.

\section{ACKNOWLEDGEMENTS}

The authors wish to express their gratitude to Consejo Nacional de Investigaciones Cientificas y Técnicas (CONICET, Argentina) for its financial support. We also thank Professor J. C. Heinrich for valuable discussion.

\section{REFERENCES}

1. O. C. Zienkiewicz, S. Qu, R. L. Taylor and S. Nakazawa, 'The patch test for mixed formulations', Int. j. num. methods eng., 23, 1873-1883 (1986).

2. G. Buscaglia, 'Numerical simulation of viscoelastic fluid flow', Ph.D. Thesis, Instituto Balseiro, San Carlos de Bariloche, 1993.

3. G. Buscaglia, 'Some experiences with several stress-velocity-pressure mixed finite elements for Newtonian flows', Int. $j$. numer. methods fluids, in press.

4. V. Girault and P. A. Raviart, Springer Series in Computational Mathematics, Vol. 5, Finite Element Methods for NavierStokes Equations. Theory and Algorithms, Springer, Berlin, 1986.

5. F. Thomasset, Implementation of Finite Element Methods for Navier-Stokes Equations, Springer, New York, 1981.

6. R. Codina, 'A finite element model for incompressible flow problems', Ph.D. Thesis, Univ. Polit. de Catalunya, Barcelona, 1992.

7. R. Vichnevetsky and J. B. Bowles, 'Fourier analysis of numerical approximations of hyperbolic equations', SIAM Stud. Appl. Math., (1982)

8. M. Storti, N. Nigro and S. Idelsohn, 'Equal-Order Interpolations: A Unified Approach to Stabilize the Incompressible and Convective Effects', Comput. Methods Appl. Mech. Eng., submitted.

9. O. C. Zienkiewicz and J. Wu, 'Incompressibility without tears-how to avoid restrictions on mixed formulations', Int. $j$. numer: methods eng., 32, 1189-1204 (1991-1992).

10. G. E. Schneider, G. D. Raithby and M. M. Yoyanovich, 'Finite element analysis of incompressible fluid flow incorporating equal order pressure and velocity interpolation', in C. Taylor, K. Morgan and C. A. Brebbia (eds), Numerical Methods in Laminar and Turbulent Flow, Pentech, Plymouth, 1978.

11. M. Kawahara and K. Ohmiya, 'Finite element analysis of density flow using the velocity correction method', Int. j. numer. methods fuids, 5, 981-993 (1985).

12. O. C. Zienkiewicz, J. Szmelter and J. Peraire 'Compressible and incompressible flow: an algorithm for all seasons', Comput. Methods Appl. Mech. Eng., 78, 105-121 (1990).

13. J. Douglas and J. Wang, 'An absolutely stabilized finite element method for the Stokes problem', Math. Comput., 52, 495508(1989).

14. T. J. R. Hughes, L. Franca and M. Balestra, 'A new finite element formulation for computational fluid dynamics: V. Circumventing the Babǔska-Brezzi condition: a stable Petrov-Galerkin formulation of the Stokes problem accommodating equal-order interpolations', Comput. Methods Appl. Mech. Eng., 59, 85-99 (1986) 
15. T. E. Tezduyar, S. Mittal, S. E. Ray and R. Shih, 'Incompressible flow computations with stabilized bilinear and linear equalorder-interpolation velocity-pressure elements', Comput. Methods Appl. Mech. Eng., 95, 221-242 (1992).

16. L. Franca and A. Madureira, 'Element diameter free stability parameters for stabilized methods applied to fluids', Comput. Methods Appl. Mech. Eng., in press.

17. S. L. Frey, L. P. Franca and R. Sampaio, 'Stabilized methods for the incompressible Navier-Stokes flow', in H. Alder et al., (eds), Numerical Methods in Engineering and Applied Sciences. 